\title{
The Notion of Community in United States Computational Science Education Initiatives
}

\author{
Mary E. Searcy and Jill T. Richie \\ Appalachian State University, Department of Mathematical Sciences \\ Boone, NC 28608
}

\begin{abstract}
The computational science education community in the United States can be perceived at a variety of levels: a community of stakeholders, educators, colleagues, or learners. Each level is illustrated by examples and their roles are discussed.
\end{abstract}

\section{Introduction}

In the last decade, addressing international problems such as communicating SARS medical information [1] and researching microgravity combustion for international space station fire prevention [2] has required computational methods. Rapid technological growth has made it possible to meet larger challenges and resolve longunanswered questions. As a consequence, there has been an increased pressure on United Stated academic institutions to prepare students for such a world [3]. As the number of computational science education initiatives increases, the notion of community - a social group sharing common characteristics or interests and perceived or perceiving itself as distinct in some respect from the larger society within which it exists [4], has become important to their development and implementation. This article explores the United States computational science education community and ways to strengthen it in the future.

\section{A Community of Stakeholders}

The United States Department of Energy's (DOE) Office of Science [5] suggested the following steps to help equip scientists for upcoming computational challenges: establish partnerships with computer vendors, strengthen existing high-end computing capability and networking, invest in advanced architecture, and collaborate with federal agencies and the academic community to find better ways to use existing and new capabilities. They advocate establishing connections among key computational science stakeholders, those individuals or agencies, with direct interest, involvement and/or investment in computational science endeavors. For example, the DOE's Scientific Discovery through Advanced Computing (SciDAC) funded projects (http://www.osti.gov/scidac/index.html) and collaborations with the United States national laboratories (http://www.scied.science.doe.gov/scied/sci_ed.htm) help support innovative partnerships between academia and other stakeholders. Also, the National Lambdarail (NLR) [6], which aims to provide a network infrastructure to 
facilitate collaborative research, could possibly be one of the most significant computational science collaborations impacting education in the future.

There are many efforts to strengthen high-end computing. The Terascale Cluster project [7] at Virginia Tech has developed one of the fastest and most inexpensive supercomputers using currently available off-the-shelf components and working with industry partners. This effort has made complex projects accessible to local educators and researchers. For those without connections to super-computing facilities and who still want to give their students experiences with parallel processing, take an open lab of Pentium computers and boot up a pre-configured distributed computing environment with the Bootable Cluster CD (http://www.cs.uni.edu/ gray/bccd/). This $\mathrm{CD}$, motivated by collaboration between educators and LinuxCare, was created solely "to facilitate instruction of parallel computing." [8]

\section{A Community of Educators}

We now shift our focus to the community of educators. Two major 2003 initiatives were the SC03 Supercomputing Conference Education Program (http://www.scconference.org/sc2003/education/edu_program.html) and the National Computational Science Institute (NCSI) (http://www.computationalscience.org/), both evaluated by the authors. Teams of educators from across the United States were chosen to spend four days learning about computational science education opportunities and technologies. The teams came from a variety of backgrounds. More than half were male (56\%); two-thirds were PhD's and 20\% were K-12 teachers. Although most participants had some experience with statistics, linear algebra, differential equations and numerical analysis, only $25 \%$ said they had advanced knowledge of calculus. They also had a variety of science knowledge in biology, chemistry, physics and computer science; $10 \%$ to $15 \%$ rated themselves as advanced in one or more of these fields. Approximately $75 \%$ said that they already incorporated modeling in their teaching, though that activity was a less common teaching strategy than traditional lecture $(96 \%)$ or hands-on activities $(97 \%)$.

Participants entered into the program with personal definitions of computational science that were as varied as their backgrounds. Here are a few quotes taken from participants' pre-survey in response to the question, "What does computational science mean to you?"

- The science of computation using sequential and parallel programming, for solving engineering and science problems

- Numbers brought together to create meaning.

- Access to ideas, tools, data, and people.

- AI, representational theory, complex systems applications and theory, automated feedback to learners, adaptive testing, responsive dissemination of online resources, ...

- It means using computers and other forms of technology to model or simulate natural systems. The model may be numeric data and equations based on the data or a visual representation using the data to generate graphics.

- problem solving

- At this point, nothing. 
Yasar and Landau [3] state that three elements have developed at the heart of defining computational science: a collection of computational tools, a collection of computational methods, and a problem-solving mindset that uses one discipline's information to solve another discipline's problems. Some elements can be seen in the definitions above; but there is a distinct lack of coherence among responses. Community, at this level, helps educate educators by providing an arena for exposure to new ideas and personal construct development.

The other current major program in the United States, NCSI, targets primarily university and college faculty. They address the community notion by partnering with universities, supercomputing facilities, non-profit foundations, and professional organizations. NCSI holds weeklong computational science education workshops where faculty are introduced to two major interpretations of computational science education [9]: 1) (computational science) education which emphasizes the modeling and visualization concepts, tools, and applications and how they should be used and 2) computational (science education) where the emphasis is on using visualization and modeling concepts, tools and applications to help students understand science ideas. In the summer of 2003, over 200 faculty were involved in workshops across the United States. NCSI participants were largely male $(64 \%)$ and white $(62 \%) ; 82 \%$ were $\mathrm{PhD}$ 's; $13 \%$ were from community colleges; $82 \%$ were from a college or university. About $95 \%$ reported that a primary reason for attending a NCSI workshop was because they were interested in using modeling and visualization in instruction. About $80 \%$ cited the desire to network with other instructors, and $73 \%$ said that they believed attendance could help facilitate changes in their home departments. Participants were encouraged to collaborate and required to produce an educational product by the week's end. After the workshops ended, participants' short-term goals included learning more about computational science and exploring new technologies, demonstrating existing models in their courses, redesigning laboratory experiences, setting up clusters, and designing new courses or new degree programs.

\section{A Community of Colleagues}

There is a critical level of community missing at many institutions, a community of colleagues on a given campus who are interested in using computational science activities in their classrooms. Support from other local faculty can be a powerful resource for instructors. At a 2003 NCSI workshop, Tennessee community college faculty from three different disciplines developed a genetics model to be used in biology courses. Another institution, with six campuses outside of Atlanta, Georgia, will begin an institution-wide computational science education initiative in 2004 . Their Center for Learning and Teaching (CLT) knows of "pockets" of faculty already involved in such activities and would like to see the development of a support network for the faculty that can be sustained over time.

\section{Future Directions}

This paper has shown there is substantial interest in computational science education; yet, the community at most levels is still somewhat fragmented. There are two primary 
suggestions the authors offer for community development. Although the notion of computational science education community can be perceived at different levels, the community that truly motivates this discussion is the community of learners. As computational science opens doors to a deeper, more dynamic, understanding of our world for researchers and industry, computational science education is opening doors to a deeper, more dynamic, understanding of course content and its applications to our world for both faculty and students. The community of learners is essential to our progress as educators and must remain the focus in this rapidly changing environment. Along with keeping this critical focus, the authors also advocate an annual computational science education conference. Conferences such as ICCS 2004 that have an education component are beneficial, particularly in bringing together stakeholders. However, a focused conference might provide a catalyst for a more cohesive notion of community in the future.

\section{References}

1. Gannis, M. (June 2003). SARS grid aids Taiwan. Access Online. Retrieved February 17, 2004 from http://access.ncsa.uiuc.edu/Stories/SARS/

2. NASA Center for Computational Science. (2002). NCCS highlights FY 2000: Enabling NASA earth and space sciences. Retrieved February 17, 2004 from http://sdcd.gsfc.nasa.gov/SCB/Highlights/FY2000/microgravity.10.html

3. Yasar, O. \& Landau, R. (2003). Elements of computational science and engineering education, SIAM Review, 45(4), 787-805.

4. Webster's Encyclopedic Unabridged Dictionary of the English Language. (1989). New York, New York: Portland House.

5. U.S. Department of Energy Office of Science. (2002). The challenge and promise of scientific computing. Retrieved February 17, 2004 from http://www.science.doe.gov/Sub/Occasional_Papers/Occasional_Papers.htm

6. The National Lambdarail, (2003). Retrieved February 17, 2004 from http://www.nationallambdarail.org/

7. Terascale cluster press release - computing.vt.edu. (2003). Retrieved February 17, 2004 from http://computing.vt.edu/research_computing/terascale/pressrelease.html

8. Gray, P. (November, 2003), Implementing computational science in the [computer science] classroom. Paper presented at the SC2003 Education Program, Phoenix, AZ.

9. Panoff, R. (July 2003). Modeling as an interactive learning environment: (Beyond PowerPoint-lessness). Paper presented at a National Computational Science Institute Workshop, Champaign, IL. 